\title{
Detection of needle tract implantation and peritoneal seeding after radiofrequency ablation using intraoperative near-infrared fluorescence system for recurrent hepatocellular carcinoma: a case report
}

Masashi Nakamura, Shinya Hayami, Masaki Ueno, Manabu Kawai, Atsushi Miyamoto, Norihiko Suzaki, Seiko Hirono, Ken-ichi Okada, Motoki Miyazawa, Yuji Kitahata and Hiroki Yamaue ${ }^{*}$ (D)

\begin{abstract}
Background: Radiofrequency ablation (RFA) for hepatocellular carcinoma (HCC) is already fully established worldwide. Needle tract implantation and peritoneal seeding occasionally occur by RFA, and the prognosis of these cases is thought to be poor. In this study, intraoperative real-time near-infrared fluorescence (NIRF) system by indocyanine green (ICG) incidentally detected both needle tract implantation and peritoneal seeding. As the utility of this system for identification of implanted and disseminated lesions after RFA for HCC has not been widely reported, we report a case of successful detection by real-time ICG-NIRF imaging and subsequent resection.

Case presentation: A 76-year-old man originally underwent medial sectionectomy for HCC in 2009. When repeated intrahepatic recurrence occurred, he underwent RFA and transcatheter arterial chemoembolization (TACE) for recurrent HCC twice at segment III and once at segment IV. In 2013, the second hepatectomy for recurrent HCC at segment VIII was performed. In 2016, he had recurrent HCC at segment III around a previous RFA and TACE scar; therefore, left lateral sectionectomy was planned. ICG-NIRF system was used to observe a main intrahepatic metastasis at segment III and to search for other tumors in the remnant liver. Although there was no signal on the surface of the remnant liver, tiny signals were observed in the abdominal wall and greater omentum. These tumors were on the needle tract of the previous RFA; both lesions, therefore, were resected. These tumors were pathologically proven to be HCC metastases. The patient has had no recurrence 14 months after the last hepatectomy.

Conclusions: ICG-NIRF system might be helpful in the detection of not only intrahepatic lesions but also needle tract implantations or peritoneal seeding. RFA should be avoided in patients with high risk of needle tract implantation and peritoneal seeding.
\end{abstract}

Keywords: Hepatocellular carcinoma (HCC), Radiofrequency ablation (RFA), Needle tract implantation, Peritoneal seeding, Indocyanine green (ICG), Near-infrared fluorescence (NIRF)

\footnotetext{
* Correspondence: yamaue-h@wakayama-med.ac.jp

Second Department of Surgery, School of Medicine, Wakayama Medical

University, 811-1 Kimiidera, Wakayama 641-8510, Japan
} 

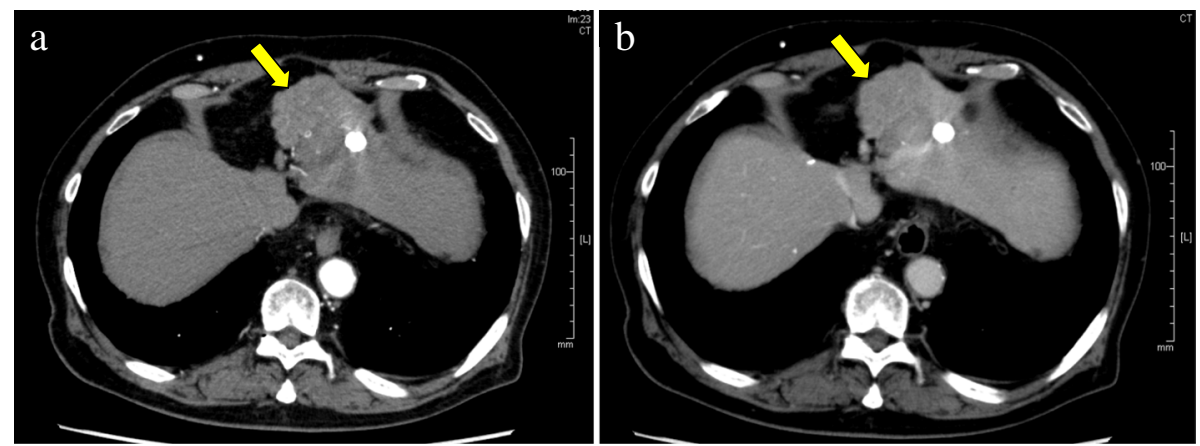

Fig. 1 Enhanced computed tomography (CT) showed a recurrent lesion with radiofrequency ablation (RFA) and transcatheter arterial chemoembolization (TACE) scar inside (30 mm in diameter) at the surface of segment III. a Early arterial phase. b Delayed phase

\section{Background}

Radiofrequency ablation (RFA) is one curative treatment option for hepatocellular carcinoma (HCC). The safety and outcomes of RFA are comparable to those of surgical treatments for small HCC. Kudo et al., on the other hand, reported the frequency of major complications after RFA as being up to $8.9 \%$ [1-3]. Needle tract implantation and peritoneal seeding after percutaneous RFA for HCC was reported by Llovet et al. [4]. Frequency of needle track seeding was reported as $12.5 \%$ (4/32 cases). Dissemination after RFA might not, however, occur at such a high frequency and it is almost absent in many reports from Japan [2]. Tumor implantation and peritoneal seeding after percutaneous RFA are considered to be a highly advanced stage and the operative indication for implanted HCC might be limited [5].

Intraoperative detection of HCC using near-infrared fluorescence (NIRF) system was reported by Gotoh et al. and Ishizawa et al. [6, 7]. This technique uses indocyanine green (ICG) and has been intraoperatively applied to liver surgery to detect novel lesions for around a decade. The usefulness of NIRF system for the detection of needle track seeding after RFA is, however, not well reported.

In this study, implanted and disseminated lesions were incidentally detected and then resected in one case using NIRF system during an operation for recurrent HCC after RFA. We present a case of real-time ICG-NIRF system being used to detect implanted and disseminated lesions.

\section{Case presentation}

A 76-year-old man originally underwent medial sectionectomy for HCC in 2009. When repeated intrahepatic recurrence occurred, he underwent RFA and transcatheter arterial chemoembolization (TACE) for recurrent HCC twice at segment III and once at segment IV. A 1.5-cm-diameter tumor at segment III was ablated by RFA needle twice for the first time. Four months after this treatment, a $1-\mathrm{cm}$ diameter of new recurrence around the previous lesion at segment III was pointed out and ablated once by RFA needle. In 2013, the second hepatectomy for recurrent HCC at segment VIII was performed. In 2016, he had recurrent HCC at segment III around a previous RFA and TACE scar again; left lateral sectionectomy was therefore planned (Fig. 1).

The patient had persistent infection of chronic hepatitis $\mathrm{C}$ and diabetes requiring insulin. Laboratory data before this operation is shown in Table 1. Focused on

Table 1 Laboratory data on admission

\begin{tabular}{|c|c|c|c|c|c|}
\hline Value & & Unit & Value & & Unit \\
\hline WBC & 61.6 & $\left(10^{2} / \mu \mathrm{l}\right)$ & Alb & 4.6 & $(\mathrm{~g} / \mathrm{dl})$ \\
\hline $\mathrm{RBC}$ & 425 & $\left(10^{4} / \mu \mathrm{l}\right)$ & AST & 20 & $(I U / I)$ \\
\hline $\mathrm{Hb}$ & 12.7 & $(g / d l)$ & ALT & 15 & $(\mathrm{IU} / \mathrm{I})$ \\
\hline $\mathrm{Ht}$ & 38.2 & (\%) & ALP & 283 & $(\mathrm{IU} / \mathrm{I})$ \\
\hline PLT & 20.9 & $\left(10^{4} / \mu \mathrm{l}\right)$ & T-bil & 0.6 & $(\mathrm{mg} / \mathrm{dl})$ \\
\hline Neu & 70.2 & (\%) & D-bil & 0.1 & $(\mathrm{mg} / \mathrm{dl})$ \\
\hline Eosin & 6.8 & (\%) & Cre & 1.48 & $(\mathrm{mg} / \mathrm{dl})$ \\
\hline Baso & 0.6 & $(\%)$ & eGFR & 36.5 & \\
\hline Mono & 5.5 & (\%) & BUN & 26 & $(\mathrm{mg} / \mathrm{dl})$ \\
\hline Ly & 16.9 & $(\%)$ & $\mathrm{Na}$ & 139 & $(\mathrm{mEq} / \mathrm{l})$ \\
\hline CRP & 0.33 & $(\mathrm{mg} / \mathrm{ml})$ & K & 4.4 & $(\mathrm{mEq} / \mathrm{l})$ \\
\hline CEA & 3.4 & (ng/ml) & PT (ratio) & 93 & (\%) \\
\hline CA19-9 & 5.6 & (UA/ml) & PT-INR & 1.03 & \\
\hline AFP & 1.9 & (ng/ml) & ICG R $\mathrm{R}_{15}$ & 8 & (\%) \\
\hline AFP-L3\% & Undetectable & $(\%)$ & $\mathrm{HA}$ & 127 & (ng/ml) \\
\hline DCP & 140 & $(\mathrm{mAU} / \mathrm{ml})$ & $\mathrm{HbA} 1 \mathrm{c}$ & 8 & (\%) \\
\hline
\end{tabular}

$W B C$ white blood cell, $R B C$ red blood cell, $H b$ hemoglobin, $\mathrm{Ht}$ hematocrit, PLT platelet, Neu neutrophil, Eo eosinophil, Baso basophil, Mono monocyte, Ly lymphocyte, CRP C-reactive protein, CEA carcinoembryonic antigen, CA19-9 carbohydrate antigen 19-9, AFP alpha-fetoprotein, AFP-L3\% lectin-reactive alpha-fetoprotein, DCP des-gamma carboxyprothrombin, Alb albumin, AST aspartate aminotransferase, $A L T$ alanine aminotransferase, $A L P$ alkaline phosphatase, $T$-bil total bilirubin, D-bil direct bilirubin, Cre creatinine, eGFR estimated glomerular filtration rate, BUN blood urea nitrogen, $\mathrm{Na}$ natrium, $K$ kalium, $P T$ prothrombin time, PT-INR international normalized ratio of prothrombin time, ICG $R_{15}$ indocyanine green excretion rate after $15 \mathrm{~min}$, $H A$ hyaluronic acid, $H b A 1 c$ glycated hemoglobin 


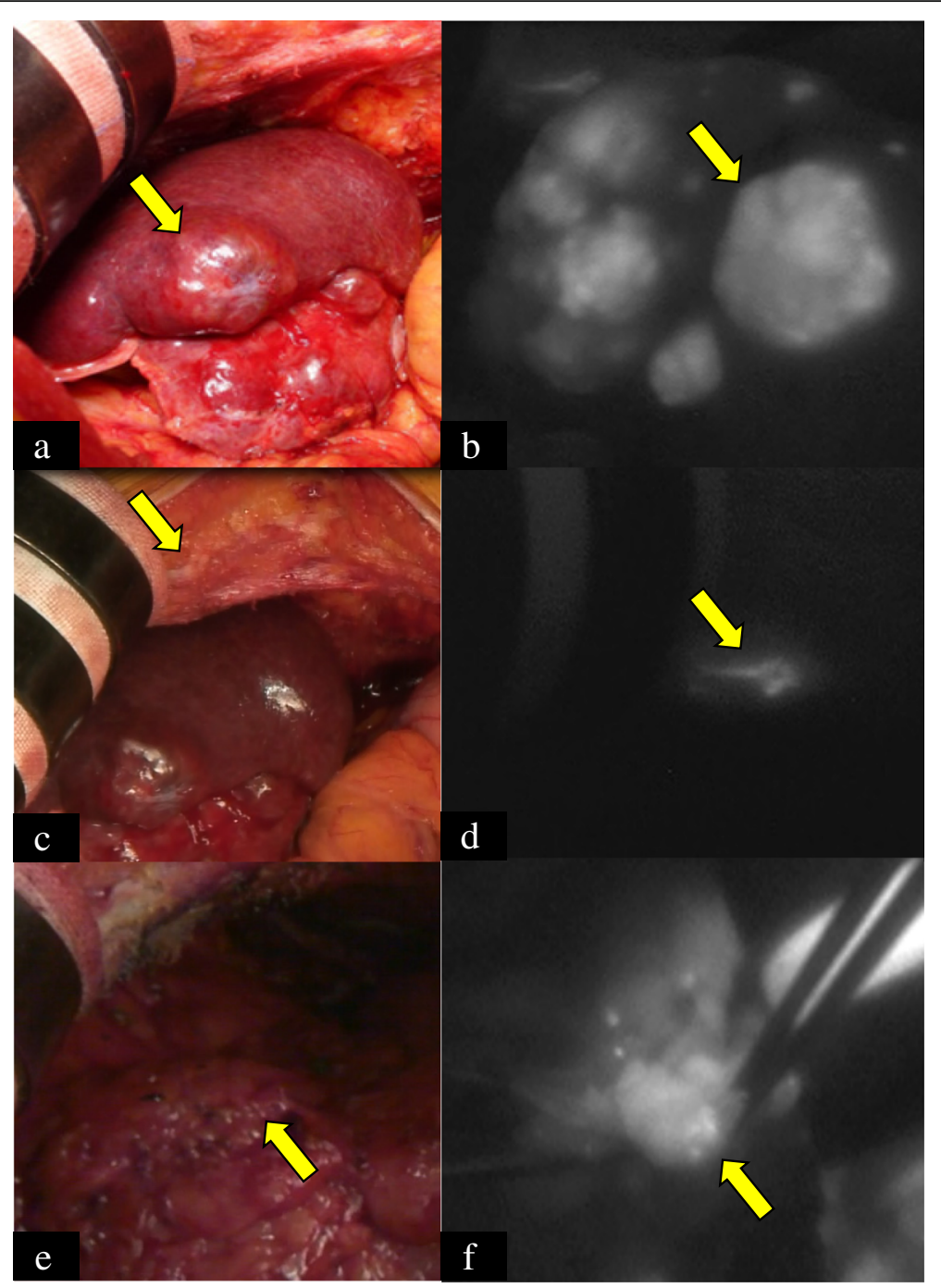

Fig. 2 Intraoperative findings using near-infrared fluorescence (NIRF) system. a Recurrent lesions were located at the surface of segment III. b Fluorescent image of the tumors at segment III. $\mathbf{c}$ A lesion of the abdominal wall was detected. $\mathbf{d}$ Fluorescent image of the abdominal wall lesion. e A lesion of the abdominal wall was detected. $\mathbf{f}$ Fluorescent image of the lesion in the greater omentum

tumor markers, des-gamma carboxyprothrombin (DCP) was high while alpha-fetoprotein (AFP) and lectin-reactive alpha-fetoprotein (AFP-L3\%) were within normal limits. Liver function was good, Child-Pugh score 5A. We therefore considered these tumors to be within operative indication for left lateral sectionectomy as the third operation on the clinical diagnosis of recurrent HCC of Union for International Cancer Control (UICC) TNM staging stage IB (T1bNOM0).

We planned to use ICG-NIRF system to observe the main intrahepatic metastasis at segment III and searched for other tumors in the remnant liver. Two days before the operation, $0.5 \mathrm{mg} / \mathrm{kg}$ ICG (Diagnogreen, Daiichi-Sankyo, Tokyo, Japan) was intravenously injected. Photodynamic eye (PDE, Hamamatsu Photonics, Hamamatsu, Japan) was used as a detector of NIRF.
Intraoperative gross appearance is shown in Fig. 2a. The recurrent tumor was located at the liver surface of segment III by fluorescent signal that could be detected by NIRF system (Fig. 2b). There was no signal on the surface of future remnant liver (right lobe). We incidentally observed tiny signals in the right upper abdominal wall, however (Fig. 2c, d). Another fluorescent tumor was detected in the greater omentum (Fig. 2e, f). These tumors were thought to be seeding of the previous RFA, but localized and controlled. After left lateral sectionectomy, they were also resected. Fluorescent signals were observed from all tumors in each specimen, even after resection (Fig. 3). Operation time was $141 \mathrm{~min}$ and intraoperative bleeding was $230 \mathrm{ml}$.

Histopathological examination revealed that the main recurrent liver tumor was moderately differentiated HCC 


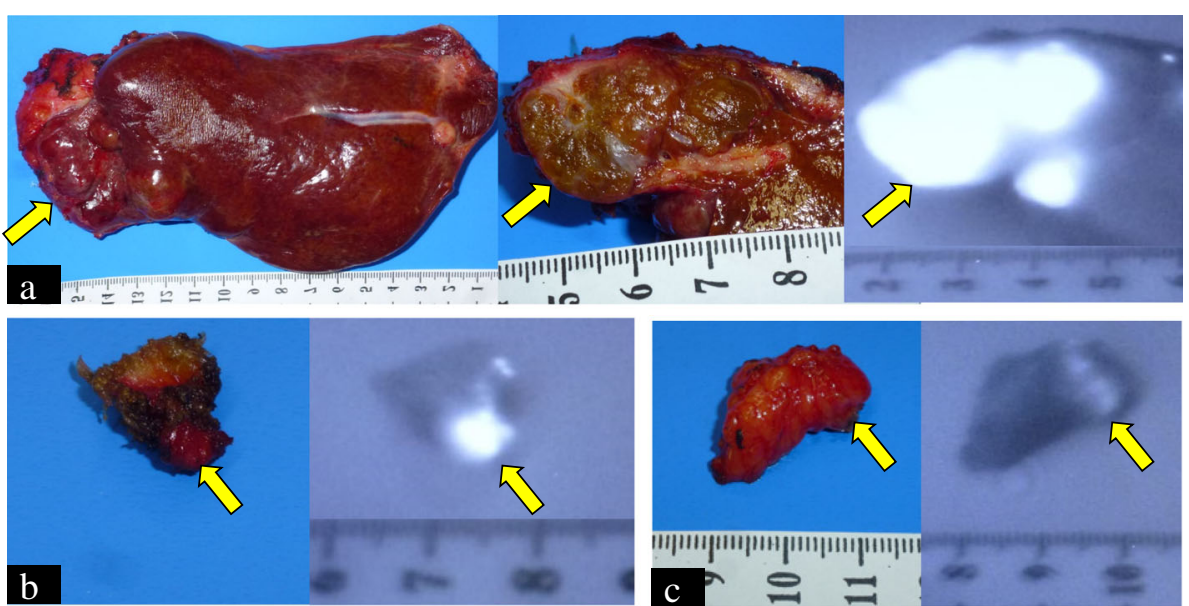

Fig. 3 Specimen findings using near-infrared fluorescence (NIRF) system. a Tumors at segment III. b Abdominal wall. c Greater omentum

showing the progression pattern of pseudoglandular type, including the bile duct with microvascular invasion for portal vein (vp1) (Fig. 4a). The main tumor included both viable and necrotic areas because of the previous TACE and RFA. Both abdominal wall and greater omentum lesions were pathologically demonstrated to be metastases of HCC (Fig. 4b, c).

The patient was discharged from our hospital on the eighth postoperative day without any complications. There has been no further recurrence 14 months after the operation.

\section{Discussion}

In this study, we incidentally detected implanted and disseminated needle tract and peritoneal seeding from previous RFA by intraoperative NIRF imaging system. Detection of intrahepatic HCC was reported by Gotoh et al. [6] and Ishizawa et al. [7]. There are several reports about HCC detection using ICG-NIRF system $[8,9]$. Moreover, this system could also be used to detect extrahepatic HCC lesions, such as in the appendix [10], lymph node, lungs, peritoneum, and adrenal glands [11]. The detailed mechanism of how extrahepatic tumor cells uptake ICG and emit fluorescence remains unclear. Satou et al. hypothesized that the extrahepatic HCC cells themselves also possess the capability to take up ICG from the blood stream. The absence of other adjacent cells or vessels, including the biliary system, may have caused the ICG retention in the metastatic lesions [11]. Further

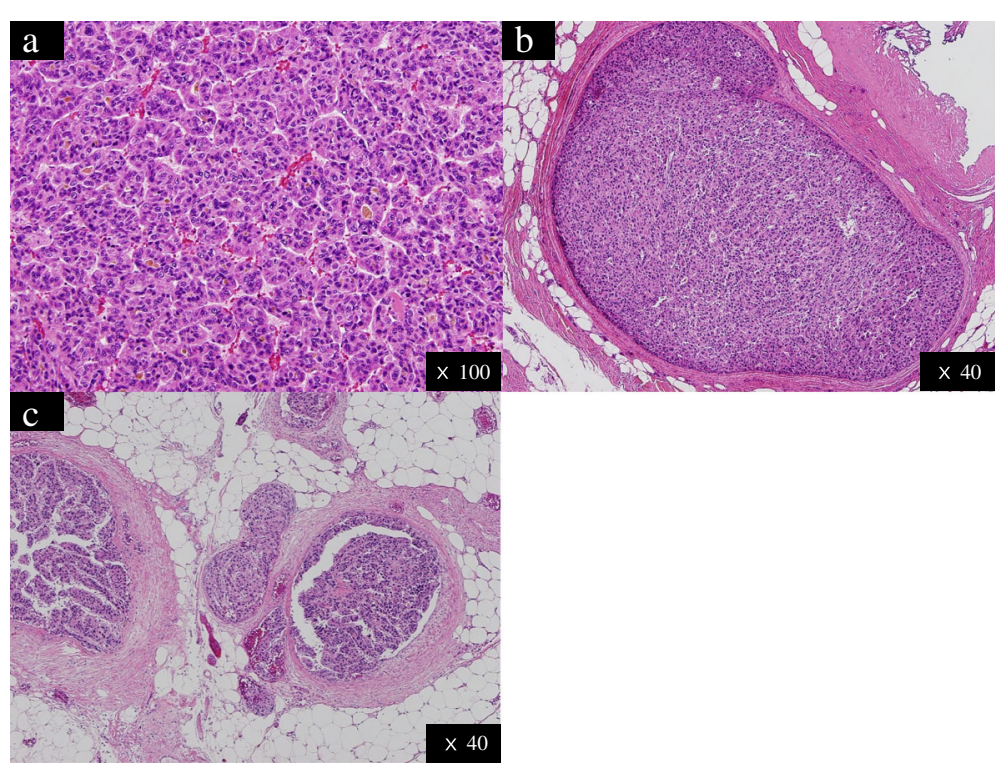

Fig. 4 Pathological images of the resected specimens. a Tumors at segment III. b Abdominal wall. c Greater omentum 
pathological examination is needed to understand this phenomenon.

This patient underwent repeated RFA for recurrent HCC. Percutaneous procedures have a risk of needle tract implantation, and peritoneal seeding and frequency of these complications are reported as between 0.9 and $12.5 \%[4,12-14]$. Llovet et al. indicated risk factors for complications include subcapsular lesions, use of cool-tip-type electrode needles, poorly differentiated HCC, and high levels of alpha-fetoprotein.

Efficacy of resection for needle tract implantation was suggested by Noda et al. [15]. One-year survival rate was $85 \%$ for patients who underwent resection of extrahepatic lesions, according to Satou et al. [11]. Operative indications are generally restricted to selective patients, however [5]. Time intervals between RFA and diagnosis of needle tract implantation were between 3 weeks and 48 months [16]; the mean doubling time was 112 (range 22-415) days [17]. ICG-NIRF system might be useful for the removal of these lesions during the operation for RFA treatment.

ICG-NIRF system has some limitations, however. The system is restricted to detection of fluorescence for tumors $5-10 \mathrm{~mm}$ from the liver surface $[8,18]$. There were also false-positive results for liver cysts and dysplastic tumors in severe liver cirrhosis [8]. In the detection of extrahepatic lesions, however, there are no such limitations. This system might be helpful in the detection of needle tract implantations or peritoneal seeding.

\section{Conclusions}

The current case study shows indication that ICG-NIRF system is helpful in the detection of needle tract implantations and peritoneal seeding as well as intrahepatic lesions.

\section{Abbreviations}

HCC: Hepatocellular carcinoma; ICG: Indocyanine green; NIRF: Near-infrared fluorescence; RFA: Radiofrequency ablation

\section{Availability of data and materials}

Data sharing is applicable to this article.

\section{Authors' contributions:}

$\mathrm{MN}, \mathrm{SH}$, and $\mathrm{MU}$ contributed in the study conception and design. MN, SH, $M U, A M$, and NS were involved in the acquisition of data. MN, SH, MU, AM, and $\mathrm{NS}$ analyzed and interpreted the data. MN, SH, and MU drafted the manuscript. $\mathrm{MK}, \mathrm{SH}, \mathrm{KO}, \mathrm{MM}, \mathrm{YK}$, and $\mathrm{HY}$ contributed in the critical revision. All authors read and approved the final manuscript.

Ethics approval and consent to participate Not applicable.

\section{Consent for publication}

Informed consent was obtained from the patient for the publication of this case report.

\section{Competing interests}

The authors declare that they have no competing interests.

\section{Publisher's Note}

Springer Nature remains neutral with regard to jurisdictional claims in published maps and institutional affiliations.

Received: 8 May 2018 Accepted: 5 July 2018

Published online: 13 July 2018

\section{References}

1. Minami Y, Kudo M. Radiofrequency ablation of hepatocellular carcinoma: a literature review. Int J Hepatol. 2011;2011:104685.

2. Kudo M. Local ablation therapy for hepatocellular carcinoma: current status and future perspectives. J Gastroenterol. 2004;39(3):205-14.

3. Bouza C, Lopez-Cuadrado T, Alcazar R, Saz-Parkinson Z, Amate JM. Metaanalysis of percutaneous radiofrequency ablation versus ethanol injection in hepatocellular carcinoma. BMC Gastroenterol. 2009;9:31.

4. Llovet JM, Vilana R, Bru C, Bianchi L, Salmeron JM, Boix L, et al. Increased risk of tumor seeding after percutaneous radiofrequency ablation for single hepatocellular carcinoma. Hepatology. 2001;33(5):1124-9.

5. Takemura N, Hasegawa K, Aoki T, Sakamoto Y, Sugawara Y, Makuuchi M, et al. Surgical resection of peritoneal or thoracoabdominal wall implants from hepatocellular carcinoma. Br J Surg. 2014;101(8):1017-22.

6. Gotoh K, Yamada T, Ishikawa O, Takahashi H, Eguchi H, Yano M, et al. A novel image-guided surgery of hepatocellular carcinoma by indocyanine green fluorescence imaging navigation. J Surg Oncol. 2009;100(1):75-9.

7. Ishizawa T, Fukushima N, Shibahara J, Masuda K, Tamura S, Aoki T, et al. Real-time identification of liver cancers by using indocyanine green fluorescent imaging. Cancer. 2009;115(11):2491-504.

8. Morita Y, Sakaguchi T, Unno N, Shibasaki Y, Suzuki A, Fukumoto K, et al. Detection of hepatocellular carcinomas with near-infrared fluorescence imaging using indocyanine green: its usefulness and limitation. Int I Clin Oncol. 2013;18(2):232-41.

9. Ishizawa T, Masuda K, Urano Y, Kawaguchi Y, Satou S, Kaneko J, et al. Mechanistic background and clinical applications of indocyanine green fluorescence imaging of hepatocellular carcinoma. Ann Surg Oncol. 2014; 21(2):440-8.

10. Imada S, Noura S, Ohue M, Shingai T, Sueda T, Gotoh K, et al. Recurrence of hepatocellular carcinoma presenting as an asymptomatic appendiceal tumor: report of a case. Surg Today. 2013;43(6):685-9.

11. Satou S, Ishizawa T, Masuda K, Kaneko J, Aoki T, Sakamoto Y, et al. Indocyanine green fluorescent imaging for detecting extrahepatic metastasis of hepatocellular carcinoma. J Gastroenterol. 2013;48(10):1136-43.

12. Takamori R, Wong LL, Dang C, Wong L. Needle-tract implantation from hepatocellular cancer: is needle biopsy of the liver always necessary? Liver Transpl. 2000;6(1):67-72.

13. Livraghi T, Lazzaroni S, Meloni F, Solbiati L. Risk of tumour seeding after percutaneous radiofrequency ablation for hepatocellular carcinoma. $\mathrm{Br}\rfloor$ Surg. 2005;92(7):856-8.

14. Imamura J, Tateishi R, Shiina S, Goto E, Sato T, Ohki T, et al. Neoplastic seeding after radiofrequency ablation for hepatocellular carcinoma. Am J Gastroenterol. 2008;103(12):3057-62.

15. Noda T, Nagano H, Murakami M, Kobayashi S, Marubashi S, Miyamoto A, et al. Surgical treatment for peritoneal seeding and needle-tract implantation of hepatocellular carcinoma after ultrasound-guided percutaneous puncture. Gan To Kagaku Ryoho. 2007;34(12):2080-3.

16. Stigliano R, Burroughs AK. Should we biopsy each liver mass suspicious for HCC before liver transplantation?-no, please don't. J Hepatol. 2005;43(4): 563-8.

17. Chang S, Kim SH, Lim HK, Lee WJ, Choi D, Lim JH. Needle tract implantation after sonographically guided percutaneous biopsy of hepatocellular carcinoma: evaluation of doubling time, frequency, and features on CT. AJR Am J Roentgenol. 2005:185(2):400-5.

18. van der Vorst JR, Schaafsma BE, Hutteman M, Verbeek FP, Liefers GJ, Hartgrink $\mathrm{HH}$, et al. Near-infrared fluorescence-guided resection of colorectal liver metastases. Cancer. 2013;119(18):3411-8. 